\title{
Nivel Socioeconómico y Brecha entre Educación Secundaria Pública y Privada en Argentina. Los Datos de PISA 2015
}

\author{
Socioeconomic Level and the Gap Between Public and Private \\ Secondary Education in Argentina. The Data from PISA 2015
}

\author{
Silvia S. Quiroz * \\ Nora L. Dari \\ Rubén A. Cervini
}

Universidad Nacional de Quilmes, Argentina

\begin{abstract}
En Argentina se ha constatado reiteradamente que los alumnos de las escuelas privadas tienen un mejor desempeño académico que los de las escuelas públicas. El objetivo de este trabajo es develar si esa brecha se explica por una mayor eficacia de las escuelas privadas o si, por el contrario, ella se debe exclusivamente a factores exógenos, tales como el origen social del alumno y la composición socioeconómica del alumnado de la escuela. Para ello, se usan los datos de PISA 2015 y se analizan los resultados de la evaluación de Ciencias, Matemática y Lectura con modelos de regresión multinivel con dos niveles: escuela y alumno. Los resultados indican que cuando se controla por este conjunto de indicadores, las diferencias entre ambos tipos de escuela se desvanecen en las tres materias evaluadas. Se concluye que tales diferencias son explicadas totalmente por factores exógenos a la escuela y que ciertos aspectos metodológicos respecto de la construcción de los indicadores utilizados tienen especial importancia para develar los determinantes de esa desigualdad. Finalmente, se discuten los resultados obtenidos en este artículo en relación a los informados por otros estudios donde la brecha pública-privada ha sido incluida en el análisis.
\end{abstract}

Descriptores: Enseñanza privada; Eficiencia de la educación; Rendimiento escolar; Enseñanza secundaria; PISA 2015.

In Argentina, it has been repeatedly observed that students from private schools achieve a better academic performance than those from public schools. The objective of this work is to reveal if this gap is explained by a greater efficiency of private schools or if it is, on the contrary, exclusively due to exogenous factors, such as the social origin of the student and the socioeconomic composition of the students in the school. For this, the data of PISA 2015 are used and the results of the assessment of Science, Mathematics and Reading are analyzed with multilevel regression models with two levels: school and student. The results indicate that when the difference between both types of school is controlled by this set of indicators, it vanishes in the three evaluated subjects. It is concluded that these differences are totally explained by exogenous factors to the school and that certain methodological aspects regarding the construction of indicators to be used, are of special importance in order to reveal the determinants of that inequality. Finally, the results obtained in this article are discussed in relation to those reported by other studies where the publicprivate gap has been analyzed.

Keywords: Private education; Educational efficiency; Academic achievement; Secondary education; PISA 2015.

*Contacto: squiroz@unq.edu.ar

ISSN: $1696-4713$

www.rinace.net/reice/

revistas.uam.es/reice
Recibido: $\quad 5$ de junio 2018

$1^{\text {a }}$ Evaluación: 29 de julio 2018

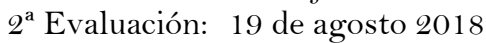

Aceptado: $\quad 3$ de septiembre 2018 


\section{Educación secundaria pública vs. privada en Argentina. Los datos de PISA 2015}

La segregación socioeconómica institucional del sistema educativo es uno de los principales mecanismos a través del cual se vulnera el principio de igualdad en la oportunidad de aprendizaje. Ello sucede cuando, en la gran mayoría de las escuelas, la composición socioeconómica del alumnado es fuertemente homogénea y esta composición es a su vez, el principal factor que explica las desigualdades en los niveles de aprendizaje de los alumnos.

Una de las formas de estructuración del sistema educativo del nivel secundario que coadyuva a ese resultado de inequidad educativa es la segmentación de la red institucional del sistema educativo en dos sectores: las escuelas de gestión estatal y las escuelas de gestión privada. Las evaluaciones de aprendizaje informan recurrentemente que los alumnos de estas últimas obtienen rendimientos promedio superiores a los de los alumnos que concurren a las escuelas estatales. Sin embargo, la existencia de inequidad educativa se corroboraría solo si tal diferencia se explicase totalmente por factores socioeconómicos. De no ser así, podría argumentarse que son diferencias en variables estrictamente institucionales o educativas (gestión, formas de organización, prácticas pedagógicas, etc.) donde se encuentran las causas de aquella brecha. Es decir, podría inferirse que las escuelas privadas enseñan mejor que las estatales y, por tanto, quedarían justificadas las políticas orientadas a aumentar el actual nivel de privatización de la educación.

Con base en los datos de PISA 2015 de Argentina, el objetivo del presente trabajo es abordar esa disyuntiva en la explicación de la brecha en los rendimientos académicos. Este objetivo tiene una relevancia particular en Argentina debido a que la participación de educación secundaria privada asciende al 30,7\%, una de las más elevadas de la Región, junto con la de Chile (SITEAL, 2013).

En el siguiente apartado se sitúa este propósito en relación con algunos antecedentes de investigación en América Latina. A seguir se expone la metodología adoptada en el tratamiento de los datos, y finalmente se presentan los resultados y las conclusiones del estudio.

\section{Antecedentes}

\subsection{La segregación social del sistema educativo}

Los sistemas de la educación primaria en América Latina están socioeconómicamente segmentados. En un análisis de los datos del Segundo y del Tercer Estudio Regional Comparativo y Explicativo (SERCE y TERCE) de la UNESCO, se ha demostrado no solo el alto poder explicativo de los factores extra-escolares (Cervini, 2012; Cervini, Dari y Quiroz, 2016; Duarte, Bos y Moreno, 2009), sino también que el mayor efecto de los indicadores del nivel socioeconómico del alumno individual se verifica en la varianza entre-escuela y no como era esperable, en la varianza intra-escuela, siendo este un fuerte indicio de segmentación socioeconómica, es decir, de una alta homogeneidad socioeconómica de los alumnos que concurren a una misma escuela. En Argentina, este mismo comportamiento se había constatado con base en más de 235.000 alumnos de $6^{\circ}$ de la educación primaria evaluados durante el Censo Nacional de Evaluación de la Calidad (Cervini, 2009). 
En un estudio reciente (Murillo y Martínez Garrido, 2017) se investigó el grado de segregación social asociado a la división del sistema de educación primaria de América Latina en público y privado. Para tal fin, fueron analizados los datos del TERCE con tres índices complementarios: índice de Disimilitud (D), índice de Inclusión Socioeconómica (IS) e índice de Raíz Cuadrada (H). Los autores concluyen que "la educación privada contribuye de forma determinante a la segregación escolar" (p. 19), entendida como "distribución desigual de los estudiantes en las escuelas en función del nivel socioeconómico y cultural de sus familias” (p. 4).

Respecto de la educación secundaria, un análisis reciente de la segregación social escolar en América Latina con los datos de PISA 2015 (Krüger, 2017) y usando tres índices (Disimilitud, Información Mutua y Aislamiento) ratifica no solo la alta segregación escolar por nivel socioeconómico en América Latina, en parte explicada por la división público/privada, sino que constata también una significativa segmentación en ambos sectores. Asimismo, otro estudio reciente (Vázquez, 2016) con los datos PISA 2015 de 72 países y utilizando varios índices de segregación (Disimilitud, Aislamiento, Raíz Cuadrada y Brecha por Centiles) constata que América Latina es una región de alta segregación escolar y llamativamente la Ciudad Autónoma de Buenos Aires y Argentina se sitúan consistentemente en el $1^{\circ}$ lugar y entre el $13^{\circ}$ y $15^{\circ}$ lugares de segregación, respectivamente. Además, el análisis de la serie PISA 2000-2015 revela al proceso de privatización como un factor relevante para entender la evolución general de esa segregación. En Argentina, un análisis multinivel de los datos del Censo Nacional de Finalización del Nivel Secundario 1998 (135.000 alumnos en 2.700 escuelas), ya había detectado también la alta homogeneidad socioeconómica del alumnado en cada escuela por el alto efecto que tienen los indicadores del nivel socioeconómico del alumno individual sobre la varianza entre-escuela (Cervini, 2005).

Todos los estudios mencionados anteriormente han utilizado bases generadas a través de evaluaciones de aprendizaje de alumnos en los niveles primario y secundario. Otros estudios, en cambio, han trabajado con encuestas de hogares y otras encuestas poblacionales en las cuales se podía determinar el tipo de escuela (público/privado), Con base en ellas, se han calculado los índices de Disimilitud, de Aislamiento y de Brecha por Centiles para medir segregación escolar público-privado en 14 países de América Latina (Arcidiácono et al., 2014) y también específicamente en Argentina (Gasparini et al., 2011). Ambos estudios concluyen que existe un alto grado de segregación escolar públicoprivado, el cual ha ido aumentando durante las últimas dos décadas. Basado en los datos de Argentina, el comienzo de este aumento se sitúa a mediados de los 1980 y y continuaba en 2010, último año incluido en el análisis.

El significativo efecto de los factores extra-escolares sobre los rendimientos académicos, la alta segmentación socioeconómica de los sistemas educativos y la superposición de la división estatal/privada con esa segmentación, características expuestas anteriormente, conceden una especial relevancia al interrogante de investigación acerca de la brecha entre los rendimientos académicos de los alumnos de los sectores público y privado.

\subsection{Público-privado: Educación primaria}

Se han realizado algunos análisis específicos sobre la distancia entre los rendimientos promedios de alumnos en los sectores público y privado de América Latina. Un estudio (Somers, McEwan y Willms, 2004) analizó con modelos multinivel los datos del Primer Estudio Internacional Comparativo (PEIC) realizado por la UNESCO en América Latina, 
encontrando que, en el conjunto de países y en cada uno de ellos, esa brecha desaparece cuando se controla por las características socioeconómicas individuales del alumno y por la composición socioeconómica de la escuela. También con modelos multinivel y la misma finalidad, Duarte, Bos y Moreno (2010) analizaron datos del TERCE y utilizaron al Índice Socioeconómico y Cultural (ISEC) individual del alumno y su promedio en la escuela como variable control. El ISEC está compuesto por a) nivel educativo del padre y b) de la madre; c) libros en el hogar; d) idioma materno; e) 21 servicios y bienes en el hogar y f) material de los pisos. Los autores constataron que la brecha estatal/privada en Matemática de $6^{\circ}$ año se mantenía significativa solo en Argentina, Costa Rica y Chile; y en Lectura solo en Chile. Sin embargo, con esos mismos datos y técnica de análisis, pero utilizando indicadores directos como bienes y libros en el hogar y educación de los padres (individuales y de 'composición' en la escuela) en vez del ISEC, tales diferencias se desvanecen totalmente (Dari, Cervini y Quiroz, 2017).

\subsection{Pública y privada: Educación secundaria}

Con los datos de PISA 2009 de Colombia, Castro, Giménez y Pérez (2014) aplican las técnicas de variables instrumentales (VI) para solucionar problemas de endogeneidad, y de descomposición de Oaxaca-Blinder (O-B) que permite identificar cuáles son los factores que explican la diferencia de resultados entre los alumnos en escuelas públicas y privadas. El análisis relaciona la comprensión lectora con ciertas características del alumno (repitencia, sexo, esfuerzo, disciplina), de la familia (libros; educación del padre y de la madre dicotomizadas) y de la escuela (pública/privada, proporción alumno/Profesor, calidad de materiales didácticos, autonomía, cantidad de matriculados). Cuando se aplica O-B a través de Mínimos Cuadrados Ordinarios (MCO), los factores observados solo explican el 43,67\% de la varianza. Cuando se aplica a través de VI, el estudio concluye que la brecha publico/privada se explica principalmente por las características del alumno, particularmente la condición de repitencia escolar, mientras que la condición socioeconómica familiar y las características de la escuela no son estadísticamente significativas. De todas formas, la brecha público-privado persiste significativa, aún después de ajustar por todas las variables consideradas.

En el mismo país, sin embargo, otro estudio (Castro, Díaz y Tobar, 2016) analizó las pruebas SABER 11/2014 ( $11^{\circ}$ de secundaria) con la técnica de O-B, contando con mediciones referidas al alumno (sexo, rezago escolar, si trabaja, repitencia y estrato socioeconómico), a la familia (años de educación de la madre, y la ocupación del padre, ingreso del hogar y el uso de tecnologías de información y comunicación en el hogar) y a la escuela (ubicación urbano/rural, y su jornada escolar), y concluyó que la diferencia entre ambos sectores se debe, en primer lugar, a las diferencias en las características de la escuela, seguido de las divergencias en el estatus socioeconómico y cultural del hogar, y en último lugar, por las diferencias en características individuales.

Con los datos de PISA 2012 de Costa Rica y aplicando también la técnica O-B, otro estudio (Giménez y Castro, 2017) concluye en cambio, que la diferencia entre escuelas públicas y privadas se explica principalmente por las características del hogar (años de estudio de la madre y del padre, índice de riqueza, índice de posesiones culturales), seguido por los recursos escolares (índice de calidad de los recursos educativos del centro, la ratio alumnos/profesor y un índice de falta de profesores), las características del estudiante (género, preescolar, repitencia) y el ambiente de trabajo de los centros (índice de relaciones profesor-alumno, la motivación del profesorado y un índice de disciplina en el centro). No 
obstante, persistiría una diferencia significativa inexplicada entre los resultados en las escuelas públicas y privadas. Con los mismos datos y cercano enfoque metodológico, Fernández y Del Valle (2013), concluyen que el año que cursa y los antecedentes de repitencia del alumno son los factores más relevantes. Aún después de considerar todas las variables de control, sin embargo, persiste una diferencia significativa entre los colegios públicos y privados, aunque "no es tan contundente". En Ciencias, el tipo de administración explica el 6\% de la variación de los resultados.

En Brasil, Oliveira, Belluzzo y Pazello (2013) aplican métodos de regresión de Quantile a los resultados de pruebas de estudiantes de $8^{\circ}$ grado y concluyen que los estudiantes de bajo rendimiento en las escuelas públicas empeorarían sus rendimientos si concurriesen a una escuela privada.

Basado en los datos de PISA 2012, un trabajo más reciente analiza las causas de las diferencias en resultados escolares entre centros educativos públicos y privados en 8 países de América Latina (Castro, Giménez y Pérez, 2017). Se utilizan las técnicas de VI en dos etapas y la descomposición de O-B. Se consideran algunas características individuales de los alumnos, de las familias, y los recursos de los centros educativos. Los autores concluyen que aún después de considerar todas las variables, los puntos promedio de diferencias fueron 67,1 en Lectura, 65,8 en Matemática y 63,8 en Ciencias. Por tanto, persiste una desigualdad muy pronunciada entre ambos sectores que no ha sido explicada por los factores considerados.

En un estudio publicado por el Banco Mundial (De Hoyos, Holland y Troiano, 2015) se analizan los datos de PISA 2006-2012 con regresiones tipo Función de Producción Educativa (FPE), incluyendo entre otras, variables relativas al nivel socioeconómico familiar, a saber: un índice de nivel económico familiar, educación de los padres y libros en el hogar. Las estimaciones de la FPE con los datos de PISA 2012 muestran una capacidad explicativa significativa del nivel socioeconómico, el género, la autonomía escolar y las calificaciones de los docentes. Al mismo tiempo, se informa que la distancia pública/privada continúa significativa al $1 \%$ aún después de controlar por todos esos factores.

En todos los estudios sobre la brecha público-privado mencionados anteriormente, no se usaron modelos multinivel sino modelos de regresión clásicos (FPE) y en algunos, las técnicas de VI y B-O, o de micro-simulación, para determinar el efecto que tienen los cambios en cada uno de los predictores sobre los resultados de aprendizaje. Las justificaciones más frecuentes para optar por tales técnicas es que permiten evaluar la existencia de endogeneidad, operación no disponible en los modelos multinivel, lo cual puede conducir a coeficientes inconsistentes y sesgados. Además, permiten determinar el aporte que realiza cada grupo de variables a la explicación de la variación de los resultados en las pruebas de rendimiento académico.

En contraposición, al no usar la técnica multinivel también los coeficientes estimados podrían ser inconsistentes y poco confiables dado que la estructura anidada de los datos viola el supuesto de aleatoriedad, según lo confirman los estudios de segmentación socioeconómica del sistema educativo. Por otro lado, el uso de indicadores-síntesis o de mediciones dicotómicas para medir nivel socioeconómico resta eficacia explicativa a este tipo de factor. Finalmente, llama la atención que, en ninguno de los estudios mencionados anteriormente, se haya incluido la composición socioeconómica del alumnado de la escuela, 
un factor ampliamente considerado por las investigaciones sobre este tema y especialmente relevante para América Latina.

En un artículo reciente (De Jorge-Moreno, 2016), los datos de PISA 2009 de Argentina, Brasil, Chile, Colombia, México, Panamá, Perú y Uruguay, son analizados con modelos multinivel para determinar el efecto del tipo de centro (público o privado) sobre los resultados en comprensión lectora. Se incluye el indicador de nivel socioeconómico construido por PISA (ESCS) y otras características del alumno individual (nativo/inmigrante, recursos educativos en el hogar, estructura familiar) y variables de la escuela (tamaño, clima del aula, recursos escolares y particularmente, el promedio de ESCS). El estudio concluye que la diferencia pública/privada continúa significativa al $1 \%$ aún después de controlar por las características del entorno familiar del alumno y de los recursos de la escuela.

Sin embargo, varios análisis multinivel relativos al nivel secundario de Argentina han mostrado que cuando se controla por el nivel socioeconómico de la escuela, la diferencia de rendimientos entre ambos sectores se desvanece. Ello fue constatado inicialmente en un antiguo análisis multinivel de los datos del Censo Nacional de Finalización del Nivel Secundario 1998 (Cervini, 2003), incluyendo alrededor de 135.000 alumnos en 2.700 escuelas, y reconfirmado en estudios más recientes con los datos de PISA/2006 (Formichella, 2011) y PISA/2009 (Formichella y Krüger, 2013; Ibáñez, 2017; Marchionni, Pinto y Vázquez, 2013).

No obstante, un reciente análisis multinivel con los datos de PISA 2015 de 10 países de América Latina (Krüger, 2017) no llega al mismo resultado. En la especificación de los modelos multinivel de dos niveles en cada país (alumno y escuela), se incluyen numerosos indicadores relativos al alumno (edad, género, asistencia al preescolar, asistencia al grado moda de su edad), a la familia (ESCS) y a la escuela (promedio de ESCS; proporción de mujeres; tamaño; localización; índice de escasez de recursos materiales; índice de actividades extracurriculares; índice de comportamiento docente; proporción de docentes con nivel educativo de Maestría; agrupamiento por habilidad; índice de selectividad para la admisión; índice de autonomía de la escuela; porcentaje de participación de los padres en actividades escolares). A pesar de ello, en Argentina, Costa Rica y Perú la brecha entre educación pública y privada se mantiene significativa al 1\%, 5\% y 10\%, respectivamente.

La revisión de esta literatura permite extraer las siguientes conclusiones relativas al efecto propio de la titularidad de la escuela: (a) cuando se utilizan regresiones de FPE, inclusive usando las técnicas de VI y O-B, se incluyan o no mediciones de la composición socioeconómica de la escuela, una porción significativa de la brecha público/privada resta sin ser explicada; (b) entre los que utilizan modelos de regresión multinivel, con mayor frecuencia se concluye que aquella brecha es principalmente explicada por la composición socioeconómica de la escuela; sin embargo, algunos de estos estudios detectan la persistencia de un efecto propio del tipo de escuela, aún después de controlar por aquella variable.

Esta última divergencia podría adjudicarse (i) a la inclusión de un solo indicador del nivel socioeconómico familiar y por ende de la escuela, y (ii) a la forma de construir el/los indicador/es. Ello porque la estrategia adoptada para medir una variable puede afectar su grado de ajuste como predictor de otra variable. 
El ESCS es un índice compuesto que resulta de aplicar el análisis de la componente principal a tres indicadores: educación de los padres, la ocupación de los padres más alta y los bienes (incluyendo libros) en el hogar y, por tanto, al estatus socioeconómico se lo trata como constructo latente. De la misma forma, un indicador de posesiones en el hogar de PISA se construye aplicando modelos basados en la Teoría de Respuesta al Ítem (TRI) a un conjunto de 25 ítems, es decir, se lo considera también como un constructo latente.

Estas construcciones (tipo Rasch), entonces, presuponen que existe una variable latente que es la causa del comportamiento de los ítems. La alteración de un ítem no altera la variable latente, a lo sumo incide en la precisión de la medición. El modelo Rasch está ampliamente extendido y no existe ningún reparo para su aplicación en el análisis de las pruebas de logro, bajo supuesto de la existencia de una variable latente: la habilidad del alumno. El Modelo de Crédito Parcial Generalizado (GPCM, siglas en inglés) también se justifica en el análisis de escalas de actitud (tipo Likert) o para pruebas de logro con ítems de respuestas múltiples ordenadas de acuerdo con las etapas del desarrollo cognitivo (Gempp et al., 2006). Ello porque el modelo de crédito parcial (MCP) es útil para analizar ítems con respuestas graduadas en más de dos niveles de respuestas parcialmente correctas, es decir, opciones que representan grados de aproximación a la respuesta correcta. El modelo evalúa y estima la probabilidad de producir cualquier respuesta parcialmente correcta sobre la base de ítems de elección múltiple ordenada.

Ahora bien, el nivel socioeconómico no puede considerarse la causa de los indicadores utilizados para su medición (por ejemplo, bienes en el hogar, nivel educativo, ingresos monetarios, etc.), sino que más bien, es el resultado del comportamiento de tales indicadores. Si un indicador cambia (por ejemplo, mayor cantidad de bienes) causará un cambio en el nivel socioeconómico, pero no necesariamente en los otros indicadores. Más aún, puede implicar el cambio de la propia definición del nivel socioeconómico. La habilidad del alumno (variable latente) es la causa de las respuestas a los ítems de una prueba, mientras que el nivel socioeconómico es el resultado o efecto de los indicadores considerados para su medición. No puede ser entendido como variable latente sino como el resultado de la composición de indicadores válidos para medir sus diferentes dimensiones. En trabajos anteriores (Cervini, 2012; Dari, Cervini y Quiroz, 2017) se han mostrado las diferencias en los resultados que se obtienen al aplicar uno u otro enfoque en el análisis del efecto de nivel socioeconómico sobre los resultados en pruebas de rendimiento.

Resumir todos los indicadores de nivel socioeconómico familiar en un solo índice, única medida a ser considerada en los diferentes modelos, satisface el principio de parsimonia y resuelve el problema de posible colinealidad. Este enfoque, sin embargo, desconoce la importancia de no perder capacidad explicativa. Si este aspecto se considera prioritario y no es de interés conocer el aporte específico de cada indicador directo de nivel socioeconómico incluido en un modelo, lo aconsejable sería incluir todos los indicadores que se consideren válidos para medir el nivel socioeconómico de la familia y de la escuela.

Con base en los datos de PISA 2015 de Argentina, el principal objetivo del presente trabajo es evaluar si el tipo de gestión de la escuela (estatal vs privada) tiene un efecto propio sobre el nivel y distribución de los aprendizajes o si, por el contrario, expresa solo el efecto de la composición socioeconómica y de los recursos escolares de la escuela. 


\section{Método}

Los criterios metodológicos adoptados para lograr el objetivo propuesto son los siguientes: (i) la técnica de regresión multinivel con dos niveles (alumno, escuela) es la más apropiada; (ii) se consideran solamente el nivel socioeconómico familiar (alumno) y la composición socioeconómica y los recursos educativos de la escuela; (iii) el nivel socioeconómico no se considera un constructo y, por tanto, se utilizan simultáneamente tres indicadores directos, en vez del ESCS de PISA; (iv) finalmente, para aumentar la confiabilidad en la medición del tipo de escuela (titularidad) se usan dos indicadores: el tipo de gestión (privada/pública) y el porcentaje de recursos provenientes del sector público.

Datos

Se analizan los datos de PISA 2015 correspondientes a Argentina. PISA sólo incluye a alumnos que tienen entre 15 años y 3 meses y 16 años y 2 meses, matriculados del séptimo grado en adelante en escuelas públicas y privadas. La selección tienes dos pasos: primero, se listan todas las escuelas con alumnos de 15 años y se selecciona una muestra aleatoria, con probabilidad de muestreo de acuerdo al tamaño; segundo, se listan los estudiantes de 15 años en cada escuela seleccionada y se selecciona una muestra aleatoria de ellos. En la Argentina, había 578.308 alumnos de 15 años matriculados del séptimo grado en adelante, los cuales fueron considerados elegibles para la muestra. Se obtuvo una tasa de respuesta muestral del 90\% (6.349 estudiantes evaluados sobre 7.016 seleccionados en el segundo paso).

Dada la alta segmentación socioeconómica del sistema educativo, a los datos perdidos en los indicadores de nivel socioeconómico familiar se les imputa el valor promedio de la escuela a la que asiste el alumno. Se excluyen las escuelas con menos de cinco alumnos para mayor estabilidad en las estimaciones. Con base en estas decisiones, se analizan datos de 6.316 alumnos en 231 escuelas.

\section{Variables}

Las variables dependientes son los resultados de las pruebas de Ciencia, Matemática y Lectura. Con estas pruebas, PISA pretende conocer las competencias, habilidades y aptitudes de los estudiantes de 15 años para analizar y resolver problemas, manejar información y enfrentar situaciones propias de la vida adulta actual.

PISA 2015 se focalizó en la evaluación de la competencia científica de los jóvenes de 15 años, es decir, qué deben conocer, valorar y saber hacer en situaciones relacionadas con la ciencia y la tecnología. El constructo se refiere a tres capacidades: (i) explicar fenómenos científicamente; (ii) evaluar y diseñar investigaciones científicas e (iii) interpretar científicamente datos y evidencias, las cuales requieren tres tipos de conocimientos: (i) contenidos; (ii) procedimientos y (iii) epistémico.

La prueba de competencia lectora de PISA consiste en textos y preguntas relacionadas con ellos. Se incluyen dos tipos de textos (textos continuos y textos discontinuos), referidos a contextos diferentes (uso privado, uso público, uso laboral y uso educativo), con base en los cuales se evalúan las siguientes competencias específicamente cognitivas: capacidad para recuperar información, interpretar un texto y reflexionar sobre su contenido. La inclusión de diferentes tipos de textos y de contextos se justifica porque PISA pretende evaluar competencias relativas a la solución de problemas de la vida práctica. 
La competencia Matemática evaluada por PISA corresponde a la capacidad para razonar, analizar y comunicar operaciones Matemáticas, implicando la capacidad de utilizar la Matemática para la solución de problemas de la vida cotidiana. Se incluyen procesos de grados crecientes de complejidad: reproducción (operaciones y cálculos simples y problemas del entorno y la rutina cotidiana); conexión (ideas y procedimientos matemáticos para la solución de problemas no tan ordinarios pero que aún incluyen escenarios familiares); reflexión, (solución de problemas complejos y desarrollo de una aproximación matemática original). Los contenidos evaluados (problemas de cantidad, espacio y forma, cambio y relaciones y probabilidad) se plantean en diferentes contextos o situaciones (personal; educativa o laboral, pública y científica, la cual implica el análisis de procesos tecnológicos o situaciones específicamente Matemáticas).

En cada prueba, PISA estima 10 "valores plausibles" (PV) para cada estudiante, los cuales son números aleatorios extraídos de la distribución de puntajes que podrían asignarse razonablemente a cada alumno, representando el conjunto de sus capacidades. Ello es necesario porque cada alumno responde a un cierto número de ítems y, a partir de ello, se estima cómo hubiera contestado en todos los restantes ítems aplicados, evaluando así las destrezas de una población. PISA recomienda usar los 10 valores plausibles separadamente y el valor de cualquier estadístico poblacional o parámetro de un modelo será el promedio de las 10 estimaciones basadas en los valores plausibles. En este artículo se ha seguido ese procedimiento para el análisis de los datos de Argentina.

Las definiciones de las variables independientes aparecen en el cuadro 1. Son las siguientes:

Variables del nivel Alumno:

- Educación de los padres

- Libros en el hogar

- Bienes en el hogar

- ESCS (Economic, Social and Cultural Status)

Variables del nivel Escuela:

- Titularidad

$\checkmark$ Tipo de gestión (pública o privada)

$\checkmark \%$ recursos públicos (\% del total de recursos de la escuela)

- Contexto socioeconómico

Promedio de bienes en el hogar

$\checkmark$ Promedio de libros

$\checkmark$ Promedio de educación de padres

- Recursos escolares

Recursos específicos de ciencia

Falta de material educativo

Con excepción del "tipo de gestión" (variable dummy), todas las variables han sido consideradas como intervalares a los fines del análisis y han sido centradas en su gran media. 
Cuadro 1. Definición de indicadores

\begin{tabular}{|c|c|c|}
\hline \multicolumn{3}{|l|}{ Nivel socioeconómico familiar } \\
\hline Bienes en el hogar (suma) & $\begin{array}{l}\text { ST011_1-12; } 16 ; \\
\text { ST012_2;_5;_7-9 }\end{array}$ & $\begin{array}{l}\text { Dicotomizados (ninguno }=0 ; \\
\text { uno o más }=1) \\
\text { O-18 (Suma de } 18 \text { bienes en el hogar) }\end{array}$ \\
\hline Libros en el hogar & STO13 & $1-6$ \\
\hline $\begin{array}{l}\text { Educación de los padres } \\
\text { (suma) }\end{array}$ & $\begin{array}{l}\text { STO05; STO06; } \\
\text { STO07; STO08 }\end{array}$ & $\begin{array}{l}\text { 2-12 (1, ninguna; } 2 \text {, prim. incompl.; } 3, \\
\text { prim. compl. o sec. incompl. } 4 \text {, sec. } \\
\text { compl.; } 5 \text {, univ. o terciario; } 6 \text {, posgrado) }\end{array}$ \\
\hline \multicolumn{3}{|l|}{ Características de la escuela } \\
\hline Tipo de gestión & $\mathrm{SCO} 13$ & Pública $=0 ;$ Privada $=1$ \\
\hline Recursos públicos (\%) & SCO16_1 & $0-100 \%$ \\
\hline $\begin{array}{l}\text { Recursos específicos de } \\
\text { ciencia }\end{array}$ & SCO59: SCIERES* & \\
\hline Falta de material educativo & $\begin{array}{l}\text { SCO 17: } \\
\text { EDUSHORT* }\end{array}$ & \\
\hline \multicolumn{3}{|l|}{ Composición socioeconómica } \\
\hline \multicolumn{3}{|c|}{ Promedio de bienes en el hogar } \\
\hline \multicolumn{3}{|c|}{ Promedio de bienes en el hogar } \\
\hline \multicolumn{3}{|l|}{ Promedio de libros } \\
\hline \multicolumn{3}{|c|}{ Promedio de educación de padres } \\
\hline
\end{tabular}

\section{Técnica de análisis}

El análisis estadístico por niveles múltiples es una técnica correlacional adecuada para analizar variaciones en las características de los individuos (por ejemplo, habilidad del alumno) que son miembros de un grupo (por ejemplo, escuela). Se trata, entonces, del análisis de mediciones integradas en una estructura anidada jerárquicamente. La técnica descompone la variación total de una variable en sus componentes (intra-escuela; entreescuela). A continuación, es posible estimar las asociaciones entre variables en esos diferentes niveles de agregación. En la parte fija del modelo se encuentran los parámetros que definen una línea promedio para todos los alumnos, la cual representa las relaciones entre el desempeño en las pruebas y los factores considerados, suponiendo que la intensidad de tales correlaciones es constante en todas las unidades de agregación (escuelas). En la parte aleatoria se estima la variación de los parámetros en cada nivel de agregación.

\section{Estrategia de análisis}

El análisis se desarrolla en dos pasos: (i) Usando el promedio de los valores plausibles, se explora un modelo completo con el objetivo de determinar qué variables mantienen significación estadística bajo control de todas las variables; (ii) Con base en los resultados del paso anterior, se estiman los siguientes modelos multinivel con cada uno de los valores plausibles:

$$
\begin{aligned}
& \checkmark \text { Vacío (sin predictor); } \\
& \checkmark \text { Titularidad de la escuela; } \\
& \checkmark \text { Socioeconómico familiar; }
\end{aligned}
$$


Socioeconómico familiar y contextual (escuela);

Socioeconómico familiar y contextual, y recursos escolares;

$\checkmark$ Titularidad y Socioeconómico familiar y contextual;

Modelo completo.

El análisis comienza con el Modelo Vacío (sin ningún predictor), el cual estima la descomposición de la varianza del rendimiento en dos niveles: alumno y escuela. Para Matemática, por ejemplo, se expresa así:

$$
\begin{aligned}
& \text { mat }_{i j}=\beta_{o j} \text { Cons } \\
& \beta_{0 i j}=\beta_{0}+\mathrm{u}_{0 j}+\mathrm{e}_{0 i j} \\
& \sigma_{\mathrm{eo}}, \mathrm{y} \sigma_{\mu 0}
\end{aligned}
$$

donde mat $\mathrm{t}_{\mathrm{ij}}$ es desempeño en Matemática del alumno $i$ en la escuela $j$; (cons) es una constante igual a $1 ; \beta_{0}$ es el desempeño promedio estimado; $e_{o j}$ es el "residuo" en el nivel alumno, para el $i$-ésimo alumno en la j-ésima escuela; $\mu_{0_{j}}$ es el residuo en el nivel escuela, para la $j$-ésima escuela. Estas dos variables $\left(\mathrm{e}_{0 i j} \mathrm{y} \mu_{0 i}\right)$ son cantidades aleatorias, con una media $=0$, no correlacionadas y con distribución normal. Por lo tanto, podemos estimar sus varianzas, indicadas como el cuadrado de $\sigma_{\mathrm{e} o}, \mathrm{y} \sigma_{\mu \circ}$, respectivamente, a partir de lo cual quedan determinados la variación total del desempeño en Matemática y los porcentajes de esa variación que se deben a las diferencias entre escuelas y entre alumnos. La primera representa principalmente, aunque no exclusivamente, el peso que tienen las características grupales de la escuela en la explicación de las variaciones totales del desempeño escolar en Matemática.

Ejemplificando con Matemática, el modelo final propuesto se expresa de la siguiente forma:

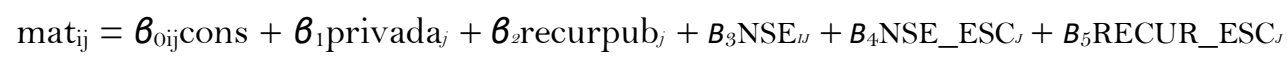

donde privada $a_{j}$ indica si la escuela $j$ es privada o no, y $\boldsymbol{b}_{1}$ la diferencia de puntaje promedio en Matemática entre escuelas públicas y privadas; recurpub $b_{j}$ es el porcentaje de los recursos de la escuela $j$ recibidos de organismos públicos y $\boldsymbol{B}_{2}$ es la intensidad de la relación con el desempeño en Matemática; $\boldsymbol{b}_{3}, \boldsymbol{b}_{4}$ y $\boldsymbol{b}_{5}$ representan la intensidad de la relación entre el desempeño en Matemática y el conjunto de indicadores del nivel socioeconómico familiar del alumno, el conjunto de indicadores de la composición socioeconómica de la escuela y los recursos escolares de la escuela, respectivamente.

En todos los casos se adopta prob. $\leq 0,001$ como criterio de significación estadística y para ello se utiliza el test de hipótesis anidada $\left(\chi^{2} \log\right.$ likelihood $)$. 


\section{Resultados ${ }^{1}$}

El análisis exploratorio inicial indicó que los tres indicadores de nivel socioeconómico familiar se sostenían estadísticamente significativos aún actuando conjuntamente. Por otro lado, el promedio de educación de los padres en la escuela resultó redundante y, por tanto, es excluido de los modelos.

\section{Efecto escuela y nivel socioeconómico familiar}

El análisis se inicia con las estimaciones de las medias globales de los desempeños en Matemática, Lectura y Ciencias y las descomposiciones proporcionales de la varianza del desempeño en las tres pruebas, sin ningún predictor (Modelo Vacío o Nulo). Las medias globales estimadas de desempeño son 431,17 en lectura, 415,26 en Matemática y 436,73 en ciencias.

La descomposición proporcional de la varianza permite estimar el efecto escuela bruto (o correlación intra-clase), entendido como la participación relativa de la variación de los rendimientos promedios de las escuelas en la variación total del rendimiento. Los resultados de esta operación se presentan en el cuadro 2. La variación debida a las desigualdades en los rendimientos promedios de las escuelas constituye alrededor de un tercio del total de la variación de los puntajes en las tres asignaturas, siendo Matemática donde se verifica la mayor variación (37\%). En contraposición, dentro de las escuelas, los alumnos tienden a diferenciarse más en ciencias. De cualquier forma, estas diferencias entre las tres asignaturas no parecen relevantes.

Cuadro 2. Descomposición de la varianza total en los dos niveles

\begin{tabular}{lccc}
\hline & CIENCIAS & MATEMÁtICA & LECTURA \\
\hline Escuela & $33,5 \%$ & $37,0 \%$ & $35,8 \%$ \\
Alumno & $66,5 \%$ & $63,0 \%$ & $64,2 \%$ \\
\hline Varianza total & 6874,03 & 6907,19 & 8269,48 \\
\hline
\end{tabular}

Fuente: Elaboración propia.

Antes de proseguir con el análisis, parece pertinente comparar la capacidad de los tres indicadores de nivel socioeconómico propuestos en este trabajo y la exhibida por ESCS, el indicador construido por PISA. Para ello, en el modelo vacío se incorporan ambas opciones alternativamente y se determina las posibles disminuciones relativas que se producen en las varianzas de ambos niveles. En el cuadro 3 se presentan los resultados de este ejercicio.

Como era esperado, todos los indicadores resultan altamente significativos, pero en todas las materias, la varianza intra-escuela explicada por los indicadores propuestos superan a la exhibida por ESCS. Estos resultados indican que los alumnos provenientes de familias con mayor "capital económico y/o cultural” tendrán mayor probabilidad de obtener mejores y más altos rendimientos en las tres asignaturas. La otra constatación relevante es que los tres indicadores propuestos exhiben una alta capacidad explicativa de la varianza entre-escuela: en las tres materias, su inclusión reduce esa varianza

${ }_{1}^{1}$ Todos los modelos procesados, con sus estimaciones y errores estándares correspondientes, pueden ser solicitados a los autores. 
notablemente, siendo la más alta la correspondiente a ciencias (36,3\%). Contrariamente, ESCS no muestra efectos sobre esa varianza.

Estos resultados, además de demostrar la conveniencia de proseguir el análisis con estos tres indicadores en vez de utilizar el índice ESCS, evidencian y reconfirman la intensa segmentación socioeconómica de la red educativa institucional informada por diversos estudios anteriores: indicadores del nivel alumno explican una porción manifiestamente abultada de la varianza en el nivel superior (escuela).

Cuadro 3. Coeficientes multinivel de indicadores socioeconómicos del alumno

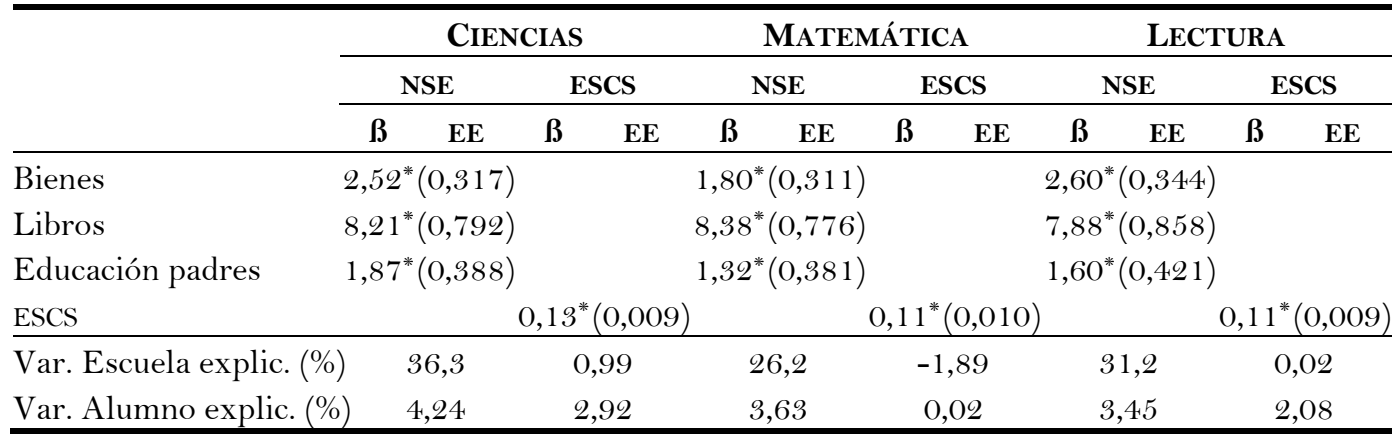

Nota: ${ }^{*}$ Prob. $\leq 0,001$.

Fuente: Elaborado por los autores.

\section{La distancia estatal-privada}

Se vuelve al Modelo Vacío, se le incorporan los dos indicadores de titularidad de la escuela y se recalculan los parámetros (cuadro 4). En las tres disciplinas existe una significativa distancia entre los rendimientos promedios de ambos sectores: los alumnos de las escuelas privadas logran puntajes significativamente más altos que los alumnos en las escuelas públicas, siendo en Lectura donde esa distancia es más notoria (superior a 51 puntos). Al mismo tiempo, se observa que "\%recursos públicos" no resulta significativo en esa misma materia. En ciencias, la titularidad de la escuela está asociada a cerca de un cuarto de la varianza total entre escuela, mientras que en las otras dos materias es levemente más reducida. Inicialmente, entonces, una proporción de las diferencias entre los rendimientos promedios de las escuelas sería explicada por diferencias vinculadas a la titularidad de la escuela. El propósito del análisis a seguir es responder al interrogante acerca de si los indicadores socioeconómicos explican totalmente esa distancia entre ambos tipos de escuela o si, por el contrario, otros factores no incluidos en los modelos o no medidos, y vinculados a la titularidad de la escuela, contribuyen a la generación de esa brecha.

Cuadro 4. Coeficientes multinivel de indicadores del tipo de escuela

\begin{tabular}{|c|c|c|c|c|c|c|}
\hline & \multicolumn{2}{|c|}{ Ciencias } & \multicolumn{2}{|c|}{ MATEMÁTICA } & \multicolumn{2}{|c|}{ LECTURA } \\
\hline & $\boldsymbol{\beta}$ & EE & B & EE & B & EE \\
\hline Privada & $43,40^{*}$ & $(7,47)$ & $40,25^{*}$ & $(8,06)$ & $51,40^{*}$ & $(8,56)$ \\
\hline \% recursos públicos & $-0,30^{*}$ & $(0,12)$ & $-0,30^{*}$ & $(0,13)$ & $-0,21$ & $(0,14)$ \\
\hline Var. Escuela explic. (\%) & \multicolumn{2}{|c|}{24,6} & \multicolumn{2}{|c|}{19,35} & \multicolumn{2}{|c|}{21,9} \\
\hline
\end{tabular}

Nota: * Prob. $\leq$ 0,001.

Fuente: Elaborado por los autores. 
Efectos del nivel socioeconómico y de los recursos escolares

Antes de responder a la pregunta anterior, parece pertinente conocer los efectos de las desigualdades socioeconómicas y de la disponibilidad de recursos escolares en la escuela. Con tal finalidad, en el modelo "vacío" se incluyen primero, a todos los indicadores del alumno y de la escuela referidos al nivel socioeconómico (Modelo 1) y a seguir, a los dos indicadores de recursos escolares institucionales (Modelo 2). Los coeficientes así recalculados se presentan en el cuadro 5. Dado que el efecto sobre la varianza del nivel alumno ya ha sido comentada anteriormente (cuadro 3), el foco de atención ahora son las modificaciones en la varianza del nivel escuela.

Cuadro 5. Coeficientes multinivel de indicadores socioeconómicos y recursos escolares

\begin{tabular}{|c|c|c|c|c|c|c|c|c|c|c|c|c|}
\hline & \multicolumn{4}{|c|}{ Ciencias } & \multicolumn{4}{|c|}{ MATEMÁTICA } & \multicolumn{4}{|c|}{ LECTURA } \\
\hline & \multicolumn{2}{|c|}{ M1 } & \multicolumn{2}{|c|}{ M2 } & \multicolumn{2}{|c|}{ M1 } & \multicolumn{2}{|c|}{ M2 } & \multicolumn{2}{|c|}{ M1 } & \multicolumn{2}{|c|}{ M2 } \\
\hline & B & EE & B & EE & B & $\mathrm{EE}$ & B & EE & B & $\mathrm{EE}$ & B & $\mathrm{EE}$ \\
\hline \multicolumn{13}{|l|}{ Familia } \\
\hline Bienes & $2,15^{*}$ & $(0,32)$ & $2,16^{*}$ & $(0,32)$ & $1,53^{*}$ & $(0,31)$ & $1,54^{*}$ & $(0,31)$ & $2,21^{*}$ & $(0,35)$ & $2,22^{*}$ & $(0,35)$ \\
\hline Libros & $7,49^{*}$ & $(0,79)$ & $7,72^{*}$ & $(0,79)$ & $8,08^{*}$ & $(0,78)$ & $8,05^{*}$ & $(0,78)$ & $7,46^{*}$ & $(0,85)$ & $7,40^{*}$ & $(0,85)$ \\
\hline Educación padres & $1,26^{*}$ & $(0,39)$ & $1,26^{*}$ & $(0,39)$ & $0,86^{*}$ & $(0,38)$ & $0,86^{*}$ & $(0,39)$ & $1, \mathrm{OO}^{*}$ & $(0,43)$ & $1, \mathrm{OO}^{*}$ & $(0,43)$ \\
\hline \multicolumn{13}{|l|}{ Escuela } \\
\hline Bienes & $7,12^{*}$ & $(1,73)$ & $5,87^{*}$ & $(1,65)$ & $5,50^{*}$ & $(2,15)$ & $4,17^{*}$ & $(2,08)$ & $10,08^{*}$ & $(2,02)$ & $8,79^{*}$ & $(1,94)$ \\
\hline Educación padres & $12,26^{*}$ & $(2,24)$ & $9,89^{*}$ & $(2,16)$ & $13,12^{*}$ & $(2,73)$ & $10,69^{*}$ & $(2,72)$ & $12,04^{*}$ & $(2,61)$ & $9,55^{*}$ & $(2,54)$ \\
\hline Recurs. de ciencia & & & $2,38^{*}$ & $(0,82)$ & & & $2,29^{*}$ & $(1,05)$ & & & $2,69^{*}$ & $(0,97)$ \\
\hline Falta de material & & & $-0,63^{*}$ & $(0,17)$ & & & $-0,70^{*}$ & $(0,22)$ & & & $-0,61^{*}$ & $(0,20)$ \\
\hline Var. Escuela (\%) & \multicolumn{2}{|c|}{13,12} & \multicolumn{2}{|c|}{11,40} & \multicolumn{2}{|c|}{21,54} & \multicolumn{2}{|c|}{19,92} & \multicolumn{2}{|c|}{15,43} & \multicolumn{2}{|c|}{13,92} \\
\hline $\begin{array}{l}\text { Var. Escuela explic. } \\
(\%)\end{array}$ & \multicolumn{2}{|c|}{71,32} & \multicolumn{2}{|c|}{75,56} & \multicolumn{2}{|c|}{54,97} & \multicolumn{2}{|c|}{59,23} & \multicolumn{2}{|c|}{68,45} & \multicolumn{2}{|c|}{72,04} \\
\hline
\end{tabular}

Nota: * Prob. $\leq 0,001$.

Fuente: Elaborado por los autores.

En el Modelo 1 (cuadro 5, M1) todos los coeficientes exhiben alta significación estadística en las tres asignaturas. De ello se infiere no solo que a mayor nivel económico y/o cultural de las familias, mayor será el desempeño esperado en las tres pruebas, sino también que, de dos alumnos con el mismo origen social familiar, aquel que concurra a una escuela de 'composición' socioeconómica más baja tendrá mayor probabilidad de obtener un desempeño más bajo en todas las materias evaluadas. Este conjunto de indicadores explica un gran porcentaje de la varianza entre-escuela de cada una de las materias, siendo en ciencia donde se observa la mayor reducción de la varianza total entre-escuela $(71,32 \%)$, implicando que ahora esa varianza representa el $13,12 \%$ de la varianza residual total del desempeño en la prueba de ciencia. La menor capacidad explicativa de este modelo se sitúa en Matemática, resultado consistente con los de diversas investigaciones: esta es la disciplina donde los factores escolares tienen el mayor impacto.

A través del Modelo 2 (M2) se pretende develar si los recursos escolares en la escuela poseen capacidad explicativa propia. Los resultados indican que, aún actuando conjuntamente con los indicadores de nivel socioeconómico, los coeficientes estimados de ambos indicadores de recursos son estadísticamente significativos en las tres asignaturas. La inclusión de estas variables aumenta la varianza entre-escuela explicada en torno de 4 puntos porcentuales. 
Titularidad de la escuela y condicionamientos del NSE

Se modelan ahora el conjunto de indicadores referidos al nivel socioeconómico y los indicadores de la titularidad de la escuela. Los resultados (cuadro 6, M3) permiten las siguientes inferencias: (i) en todas las materias, el coeficiente de 'privada' disminuye abruptamente, tornándose no significativo; la brecha pública-privada se desvanece, es decir, se explica totalmente por los condicionamientos socioeconómicos familiares y contextuales de la escuela; (ii) ‘\% recursos públicos’ mantiene una leve significación en ciencias (5\%); (iii) los coeficientes de todos los indicadores de nivel socioeconómico mantienen su nivel de significación estadística.

Modelo completo: titularidad, NSE y recursos escolares.

Con la finalidad de despejar la duda sobre \% recursos públicos, al modelo anterior se le agregan las variables de recursos escolares (cuadro 6, M4) y se constata que el coeficiente estimado para aquel indicador se torna no significativo. El resto de los coeficientes estimados en este modelo son muy similares a los estimados en el Modelo 2 (cuadro 5).

Cuadro 6. Modelos multinivel 3 y 4 de todos los indicadores

\begin{tabular}{|c|c|c|c|c|c|c|c|c|c|c|c|c|}
\hline & \multicolumn{4}{|c|}{ Ciencias } & \multicolumn{4}{|c|}{ MATEMÁTICA } & \multicolumn{4}{|c|}{ LECTURA } \\
\hline & \multicolumn{2}{|c|}{ M3 } & \multicolumn{2}{|c|}{ M4 } & \multicolumn{2}{|c|}{ M3 } & \multicolumn{2}{|c|}{ M4 } & \multicolumn{2}{|c|}{ M3 } & \multicolumn{2}{|c|}{ M4 } \\
\hline & B & EE & B & EE & $\beta$ & EE & B & EE & B & EE & B & EE \\
\hline \multicolumn{13}{|l|}{ Familia } \\
\hline Bienes & $2,15^{*}$ & $(0,32)$ & $2,16^{*}$ & $(0,32)$ & $1,53^{*}$ & $(0,31)$ & $1,54^{*}$ & $(0,31)$ & $2,21^{*}$ & $(0,35)$ & $2,22^{*}$ & $(0,35)$ \\
\hline Libros & $7,77^{*}$ & $(0,79)$ & $7,71^{*}$ & $(0,79)$ & $8,07^{*}$ & $(0,78)$ & $8,04^{*}$ & $(0,77)$ & $7,45^{*}$ & $(0,85)$ & $7,39^{*}$ & $(0,85)$ \\
\hline $\begin{array}{l}\text { Educación } \\
\text { padres }\end{array}$ & $1,26^{*}$ & $(0,39)$ & $1,26^{*}$ & $(0,39)$ & $0,86^{*}$ & $(0,38)$ & $0,86^{*}$ & $(0,38)$ & $1,00^{*}$ & $(0,43)$ & $1, \mathrm{OO}^{*}$ & $(0,43)$ \\
\hline \multicolumn{13}{|l|}{ Escuela } \\
\hline Privada & 8,32 & $(5,28)$ & 6,22 & $(4,99)$ & 7,95 & $(6,66)$ & 5,69 & $(6,42)$ & 11,87 & $(6,14)$ & 9,85 & $(5,87)$ \\
\hline $\begin{array}{l}\text { \% Recursos } \\
\text { públicos }\end{array}$ & $0,02^{*}$ & $(0,08)$ & 0,04 & $(0,08)$ & 0,01 & $(0,10)$ & 0,02 & $(0,10)$ & 0,15 & $(0,09)$ & 0,17 & $(0,09)$ \\
\hline Bienes & $6,89^{*}$ & $(1,74)$ & $5,76^{*}$ & $(1,65)$ & $5,44^{*}$ & $(2,16)$ & $4,04^{*}$ & $(2,09)$ & $9,98^{*}$ & $(2,01)$ & $8,80^{*}$ & $(1,93)$ \\
\hline $\begin{array}{l}\text { Educación } \\
\text { padres }\end{array}$ & $11,44^{*}$ & $(2,31)$ & $9,42^{*}$ & $(2,22)$ & $12,24^{*}$ & $(2,80)$ & $10,19^{*}$ & $(2,80)$ & $11,54^{*}$ & $(2,68)$ & $9,38^{*}$ & $(2,59)$ \\
\hline $\begin{array}{l}\text { Recursos de } \\
\text { ciencia }\end{array}$ & & & $2,41^{*}$ & $(0,82)$ & & & $2,31^{*}$ & $(1,05)$ & & & $2,77^{*}$ & $(0,96)$ \\
\hline $\begin{array}{l}\text { Falta de } \\
\text { material }\end{array}$ & & & $-0,61^{*}$ & $(0,17)$ & & & $-0,68^{*}$ & $(0,22)$ & & & $-0,59^{*}$ & $(0,20)$ \\
\hline $\begin{array}{l}\text { Var. Escuela } \\
(\%)\end{array}$ & 12 & & & 32 & 21, & ,39 & 19, & 83 & &, 10 & & ,60 \\
\hline $\begin{array}{c}\text { Var. Escuela } \\
\text { explic. }(\%)\end{array}$ & 71, & & 75 & ,76 & 55 & ,39 & 59 , & 45 & &, 27 & & ,79 \\
\hline
\end{tabular}

Nota: ${ }^{*}$ Prob. $\leq 0,001$.

Fuente: Elaborado por los autores.

\section{Conclusiones}

Las diferencias entre los rendimientos promedios obtenidos por los alumnos de escuelas privadas y de escuelas públicas en las pruebas de Ciencias, Matemática y Lectura aplicadas por PISA 2015 en Argentina, fueron analizadas en relación con el nivel socioeconómico de la familia del alumno, la 'composición' socioeconómica del alumnado de la escuela y los recursos educativos disponibles en la escuela. El principal interrogante del estudio fue si 
aquella brecha se debía a los factores mencionados o si, por el contrario, otras variables serían parte también de la explicación.

Tres criterios metodológicos caracterizan al análisis realizado. En primer lugar, los datos se analizaron con modelos de regresión multinivel; en segundo lugar, para la medición del nivel socioeconómico se construyeron tres indicadores específicos, en vez de usar el indicador propuesto por PISA (ESCS), por considerar que de esta forma se obtendría mayor capacidad explicativa; finalmente, se incluyeron solamente indicadores de nivel socioeconómico y recursos disponibles en la escuela.

El análisis de los datos demostró que la brecha entre los rendimientos promedios de ambos sectores se explica totalmente por diferencias en el nivel socioeconómico y en la dotación de recursos de la escuela, y no por otras características de la institución escolar, tales como formas de organización, práctica pedagógica, cultura institucional. Esta conclusión es convergente con los hallazgos informados por otras investigaciones basadas tanto en anteriores evaluaciones de PISA (educación secundaria), como las realizadas por UNESCO (educación primaria).

Estos resultados contradicen a las conclusiones de estudios basados en la aplicación de modelos de regresión clásicos (FPE) y en las técnicas de VI y B-O, expuestos en la revisión de la literatura, estudios que, además, no han incluido la composición socioeconómica del alumnado de la escuela, un factor clave en la respuesta a la pregunta central de investigación. Por otro lado, la mayoría de los estudios revisados que han utilizado modelos de regresión multinivel son convergentes con la principal conclusión en este trabajo: la brecha pública/privada se explica por la composición socioeconómica de la escuela. Sin embargo, un estudio reciente (Krüger, 2017), aplicando regresiones multinivel y empleando solamente al índice ESCS de PISA como indicador de nivel socioeconómico, constata la persistencia de aquella brecha en Argentina. El presente trabajo demuestra que la ampliación del número de ese tipo de indicadores y la renuncia al uso del índice ESCS, resulta en la explicación de la totalidad de la distancia entre sector público y privado en Argentina.

Su principal implicación política es que la expansión del sector privado, promovida bajo el argumento de su mayor eficacia para el logro de los aprendizajes, carece de fundamento empírico. Las distancias de logros se deben a las diferencias de los alumnados ('composición') de ambos tipos de escuelas y, por tanto, no es sino la forma que adquiere el proceso de segmentación socioeconómica del sistema educativo. Tal constatación indica la necesidad de dar primacía a políticas sociales gubernamentales focalizadas en el sector educativo público que sean eficaces para suavizar la incidencia de los condicionamientos socioeconómicos y culturales sobre el aprendizaje escolar. A esta misma conclusión se había arribado para el nivel de educación primaria con los datos del SERCE y del TERCE de UNESCO.

Es importante enfatizar que la selección de la metodología a ser aplicada en el análisis es una decisión de gran importancia puesto que ella frecuentemente explica variaciones de inferencias a partir de los mismos datos. Así, por ejemplo, en la publicación del Banco Mundial ya referida (De Hoyos, Holland y Troiano, 2015), los datos de la serie PISA 20002012 se analizan con una regresión del tipo FPE y un modelo de microsimulación y, sobre todo, sin incluir algún indicador referido a la composición socioeconómica del alumnado de la escuela. Sobre esta base, el estudio concluye que el aumento importante de recursos destinados a la educación pública, no ha tenido efectos positivos, ilustrado por el hecho de 
que "los resultados del aprendizaje en las escuelas públicas disminuyeron en comparación con las escuelas privadas", infiriéndose la "ineficacia de las políticas educativas basadas en insumos". El interrogante que surge es acerca de la sustentabilidad de esta conclusión cuando el análisis de esos mismos datos con regresiones multinivel que incluyen el nivel socioeconómico familiar y del alumnado de la escuela, arroja como resultado que la brecha pública-privada es completamente explicada por tales factores extra-escolares.

Los modelos aplicados no han conseguido eliminar completamente los efectos de las heterogeneidades del nivel escuela no incluidos o inobservados. De los residuos totales iniciales del nivel escuela, resultantes de aplicar los modelos multinivel a las escuelas argentinas participantes de PISA 2015 en cada una de las materias evaluadas, restan por explicarse 11,3\%, 19,8\% y 13,6\% en Ciencias, Matemática y Lectura, respectivamente. No obstante, ninguno de ellos puede ser adjudicado a presuntas características propiamente escolares o de gestión institucional de las escuelas del sector privado.

\section{Referencias}

Arcidiácono, M., Cruces, G., Gasparini, L., Jaume, D., Serio, M., y Vázquez, E. (2014). La segregación escolar público-privada en América Latina. Santiago de Chile: CEPAL.

Castro, G., Díaz, M. y Tobar, J. (2016). Causas de las diferencias en desempeño escolar entre los colegios públicos y privados: Colombia en las pruebas SABER11/2014. Recuperado de https://www.javerianacali.edu.co/sites/ujc/files/node/fielddocuments/field_document_file/ddtn262016.pdf

Castro, G., Giménez, G. y Pérez, D. (2014). El desempeño educativo escolar en Colombia: Factores que determinan la diferencia en rendimiento académico entre las escuelas públicas y privadas. Investigaciones de Economía de la Educación, 9, 895-921.

Castro, G., Giménez, G. y Pérez, D. (2017). Desigualdades educativas en América Latina, PISA 2012: Causas de las diferencias en desempeño escolar entre los colegios públicos y privados. Revista de Educación, 376(2), 33-61.

Cervini, R. (2003). Diferencias de resultados cognitivos y no-cognitivos entre estudiantes de escuelas públicas y privadas en la educación secundaria de Argentina: Un análisis multinivel. Education Policy Analysis Archives, 11(5), 5-32.

Cervini, R. (2005). The relationship between school composition, school process and mathematics achievement in secondary education in Argentina. International Review of Education, 51(2), 173-200.

Cervini, R. (2009). Class, school, municipal, and state effects on mathematic achievement in argentina: A multilevel analysis. School Effectiveness and School Improvement, 20(2), 319-340. https://doi.org/10.1080/09243450802664404

Cervini, R. (2012). El “efecto escuela” en países de América Latina: Re-analizando los datos del SERCE. Archivos Analíticos de Políticas Educativas, 20(39), 1-24.

Cervini, R., Dari, N. y Quiroz, S. (2016). Las determinaciones socioeconómicas sobre la distribución de los aprendizajes escolares. Los datos del TERCE. REICE. Revista Iberoamericana sobre Calidad, Eficacia y Cambio en Educación, 14(4), 61-79.

Dari, N., Cervini, R. y Quiroz, S. (2017, septiembre). Educación primaria pública vs. privada. Los datos del TERCE. Comunicación presentada en el VI Congreso Nacional e Internacional de Estudios Comparados en Educación. SAECE, Sociedad Argentina de Estudios Comparados en Educación, Buenos Aires. 
De Hoyos, R., Holland, P. y Troiano, S. (2015). Understanding the trends in learning outcomes in Argentina, 2000 to 2012. Washington, DC: World Bank Group. https://doi.org/10.1596/1813-9450-7518

De Jorge-Moreno, J. (2016). Factores explicativos del rendimiento escolar en Latinoamérica con datos PISA 2009. Revista de Métodos Cuantitativos para la Economía y la Empresa, 22, 216 229.

Duarte, J., Bos, M. y Moreno, M. (2009). Inequidad en los aprendizajes escolares en Latinoamérica. Lima: BID.

Duarte, J., Bos, M. y Moreno, M. (2010). ¿Enseñan mejor las escuelas privadas en América Latina? Estudio comparativo usando los resultados del SERCE. Lima: BID.

Fernández, A. y Del Valle, R. (2013). Desigualdad educativa en Costa Rica: La brecha entre estudiantes de colegios públicos y privados. Análisis con los resultados de la evaluación internacional PISA. Revista CEPAL, 117, 37-57. https://doi.org/10.18356/38309458-es

Formichella, M. (2011). ¿Se debe el mayor rendimiento de las escuelas de gestión privada en la Argentina al tipo de administración? Revista CEPAL, 105, 151-166.

Formichella, M. y Krüger, N. (2013). El fracaso escolar en el nivel medio argentino: ${ }_{\mathrm{C}}$ Es menos frecuente en las escuelas de gestión privada debido a su administración? Regional and Sectoral Economic Studies, 13(3), 127-144.

Gasparini, L., Jaume, D., Serio, M. y Vázquez, E. (2011). La segregación escolar en Argentina. Reconstruyendo la evidencia. Desarrollo Económico, 51(202), 189-219.

Gempp, R., Denegri, M., Caprile, C., Cortés, L., Quesada, M. y Sepúlveda, J. (2006). Medición de la alfabetización económica en niños: Oportunidades diagnósticas con el modelo de crédito parcial. Psykhe, 15(1), 37-51. https://doi.org/10.4067/S0718-22282006000100002

Giménez, G. y Castro, G. (2017). ¿Por qué los estudiantes de colegios públicos y privados de Costa Rica obtienen distintos resultados académicos? Perfiles Latinoamericanos, 25(49), 195-223. https://doi.org/10.18504/pl2549-009-2017

Ibáñez, M. (2017). Inclusión y equidad: Un análisis con base en el acceso y logros para el nivel medio de educación en Argentina. Semestre Económico, 20(43), 111-138. https://doi.org/10.22395/seec.v20n43a5

Krüger, N. (2017, noviembre). El panorama de la segregación social escolar en América Latina según PISA 2015. Conferencia presentada en la LII Reunión Anual de la AAEP. Universidad Nacional de La Plata, Buenos Aires.

Marchionni, M., Pinto, F. y Vázquez, E. (2013). Determinantes de la desigualdad en el desempeño educativo en la Argentina. Anales de la Asociación Argentina de Economía Política, 48, 17-36

Murillo, F. y Martínez-Garrido, C. (2017). Segregación social en las escuelas públicas y privadas en América Latina. Educação e Sociedade, 38(140), https://doi.org/10.1590/es0101-73302017167714.

Oliveira, P. R., Belluzzo, W. y Pazello, E. T. (2013). The public-private test score gap in Brazil. Economics of Education Review, 35(3), 120-133. https://doi.org/10.1016/j.econedurev.2013.04.003

SITEAL. (2013). Cobertura relativa de la educación pública y privada en América Latina. Buenos Aires: IIPE-Unesco.

Somers, M., McEwan P. y Willms, J. (2004). How effective are private schools in Latin America? Comparative Education Review, 48(1), 48-69. https://doi.org/10.1086/379841 
Vázquez, E. (2016). Segregación escolar por nivel socioeconómico: Midiendo el fenómeno y explorando sus determinantes. Económica, 62, 121-184.

\section{Breve CV de los autores}

\section{Silvia Quiroz}

Licenciada en Educación (UNQ-1998) y Magíster en Metodología de la Investigación Científica (UNLa-2009). Se desempeña como docente de la Universidad Nacional de Quilmes; dicta clases de Metodología de la Investigación Social en Grado y de Análisis de Datos Cuantitativos y Taller de Tesis en Posgrado (UVQ-UNQ). Es profesora del Taller de Trabajo Integrador Final en el Instituto Universitario de Gendarmería Nacional Argentina (IUGNA). Investiga temas vinculados al rendimiento académico en distintos niveles del sistema educativo argentino, tomando como herramienta central para el desarrollo de los estudios datos secundarios, obtenidos de bases de diversos operativos de evaluación; posee publicaciones en revistas nacionales e internacionales con referato académico. ORCID ID: https://orcid.org/OOOO-OOO2-6339-8947. Email: squiroz@unq.edu.ar

\section{Nora Dari}

Licenciada en Educación por la Universidad Nacional de Quilmes y doctoranda en el Programa Interinstitucional de Doctorado en Educación (PIDE), de las universidades UNTREF, UNLA y UNSAM. Es docente de grado y posgrado en universidades públicas y privadas a nivel nacional e internacional, investigadora Cat. III en el Programa Nacional de Incentivos a la Investigación. Se desempeña como Directora de Relaciones Internacionales en la UNQ y como Asesora Pedagógica en varias Universidades argentinas. Fue Consejera de Estudios de la Maestría en Cs Sociales, Orientación Investigación y Evaluación Educativa. Coordinadora de la Red ALCUEMOVE - Unión Europea y de proyectos birregionales (Africa- Sudamérica) del Ministerio de Educación de la Nación. Co-directora proyecto LAB-FRA, MinCyT. ORCID ID: https://orcid.org/oOOO-0002-6685-3992.Email: noradari@gmail.com

\section{Rubén Cervini}

Licenciado en Ciencias Sociales, Universidad Nacional de Cuyo, Argentina; Master en Ciencias Políticas de la Facultad Latinoamericana de Ciencias Sociales (FLACSO) y Master en Administración Educacional, Universidad del Valle/OEA, Colombia. Ha sido Profesor-investigador en universidades de Argentina, Chile, Colombia y México, y consultor de UNESCO y UNICEF en diversos proyectos. Actualmente es Director de la Maestría en Educación de la Universidad Nacional de Quilmes (Argentina) y participa en un Programa de investigación. Los resultados de sus investigaciones han sido publicados en diversas revistas de investigación educativa. Centra su trabajo de investigación en los factores escolares y extra-escolares del rendimiento escolar. ORCID ID: https://orcid.org/OOOO-0002-9307-7263. Email: racervini@fibertel.com.ar 